\title{
Targeted Gene Knockouts by Protoplast Transformation in the Moss Physcomitrella patens
}

\author{
Lei Zhu *
}

Department of Botany and Plant Sciences, University of California, Riverside, CA, United States

Targeted gene knockout is particularly useful for analyzing gene functions in plant growth, signaling, and development. By transforming knockout cassettes consisting of homologous sequences of the target gene into protoplasts, the classical gene targeting method aims to obtain targeted gene replacement, allowing for the characterization of gene functions in vivo. The moss Physcomitrella patens is a known model organism for a high frequency of homologous recombination and thus harbors a remarkable rate of gene targeting. Other moss features, including easy to culture, dominant haploidy phase, and sequenced genome, make gene targeting prevalent in Physcomitrella patens. However, even gene targeting was powerful to generate knockouts, researchers using this method still experienced technical challenges. For example, obtaining a good number of targeted knockouts after protoplast transformation and regeneration disturbed the users. Off-target mutations such as illegitimate random integration mediated by nonhomologous end joining and targeted insertion wherein one junction on-target but the other end off-target is commonly present in the knockouts. Protoplast fusion during transformation and regeneration was also a problem. This review will discuss the advantages and technical challenges of gene targeting. Recently, CRISPR-Cas9 is a revolutionary technology and becoming a hot topic in plant gene editing. In the second part of this review, CRISPR-Cas9 technology will be focused on and compared to gene targeting regarding the practical use in Physcomitrella patens. This review presents an updated perspective of the gene targeting and CRISPR-Cas9 techniques to plant biologists who may consider studying gene functions in the model organism Physcomitrella patens.

This article was submitted Genome Editing in Plants, a section of the journal

Frontiers in Genome Editing

Received: 01 June 2021 Accepted: 30 November 2021 Published: 17 December 2021

Citation:

Zhu L (2021) Targeted Gene Knockouts by Protoplast

Transformation in the Moss

Physcomitrella patens.

Front.Genome Ed. 3:719087. doi: 10.3389/fgeed.2021.719087

Keywords: Gene Targeting, Homologous Recombination, Protoplast Transformation, CRISPR-Cas9, Knockouts, Physcomitrella patens

\section{INTRODUCTION}

Gene targeting mediated by homologous recombination, widely applied in mouse embryonic stem cells, is particularly useful for studying gene functions. This invention won the Nobel Prize in physiology and medicines in 2007 (Capecchi, 2005a). In plants, the moss Physcomitrella patens is the only species harboring high gene targeting efficiency; therefore, gene targeting in Physcomitrella has been extensively used (Schaefer and Zryd, 1997). Since the first successful moss knockouts were obtained, thousands of publications emerged from adapting gene targeting to the Physcomitrella research (Girke et al., 1998; Rensing et al., 2020). 
CRISPR-Cas9 won the Nobel Prize in 2020, is a revolutionary technology, and has become a hot topic in plant and animal research to study gene functions and create variants for breeding (Ran et al., 2013; Hsu et al., 2014; Knott and Doudna, 2018). One advantage of CRISPRCas9 is that it can make marker-free knockouts suitable for generating transgene-free plants or animals. In plants, CRISPR-Cas9 has been used in species including model organism Arabidopsis and commercial plants such as tobacco, maize, soybean, and wheat (Jiang et al., 2013; Nekrasov et al., 2013; Shan et al., 2013; Liang et al., 2014). Recently, researchers would like to take advantage of CRISPRCas9 to study gene functions in Physcomitrella patens.

This review will not cover all the applications of the powerful technologies but rather focus on the practical use of successfully targeted knockouts resulting from traditional gene targeting and CRISPR-Cas9 technology.

\section{OVERVIEW OF GENE TARGETING IN PHYSCOMITRELLA PATENS}

Targeted gene knockout for generating loss-of-function alleles is essential in genome editing, allowing for the precise characterization of gene functions in cell growth, organism development, and physiological processes. Thus, many genome editing tools have been deployed to obtain knockouts, including nucleases such as zinc-finger nucleases (ZFNs), transcription activator-like effector nucleases (TALENs), RNA-guided CRISPR-Cas family, and gene targeting mediated by endogenous homology-directed repair (HDR) (Capecchi, 2005b; Miller et al., 2007; Sander et al., 2011; Wood et al., 2011; Cong et al., 2013; Knott and Doudna, 2018). In addition, gene targeting utilizes knockout constructs to target genes through homologous recombination (Schaefer, 2001; Ermert et al., 2019).

Gene targeting is widely applied in mice, yeast, and other organisms (Gardner and Jaspersen, 2014; Gerlai, 2016). In the yeast Saccharomyces cerevisiae, the predominant mechanism for repairing double-strand break (DSB) is homologous recombination. Thus, yeast exhibits high efficiency of gene targeting. The mouse is another model organism traditionally deployed to study gene knockouts, in which researchers can use precise genome editing tools (Boube and Okkenhaug, 2013). However, nonhomologous end joining (NHEJ) is the dominant pathway to repair double-strand breaks in most plants and mammals. In those organisms, foreign DNA fragments are inserted into the genome illegitimately. While most plants exhibit a very low efficiency of homologous recombination (less than 1\%), Physcomitrella patens is the only plant species exhibiting high efficiency (up to $90 \%$ ) of homologous recombination, resulting in a high ratio of targeted integration to illegitimate insertions into moss genome (Schaefer and Zryd, 1997; Quatrano et al., 2007). Homologous recombination in Physcomitrella patens is dependent on RAD51 to repair DSBs (Markmann-Mulisch et al., 2007; Schaefer et al., 2010).
Additionally, many genes have been functional in Physcomitrella patens as enhancers or repressors in the homologous recombination-mediated DSB repair (Kamisugi et al., 2012; Kamisugi et al., 2016; Wiedemann et al., 2018; Guyon-Debast et al., 2019).

The moss Physcomitrella patens belonging to the Funariaceae family of the Phylum Bryophyta is an increasingly popular model organism for studying plant evolution, development, and growth (Wood et al., 2000; Cove, 2005; Vidali et al., 2009). The Gransden strain of Physcomitrella patens was established in the United Kingdom and is now the most prevalent system used for genetic engineering in the laboratory (Engel, 1968). This nonvascular early land plant was sequenced in 2007 (Rensing et al., 2008), revealing a genome size of $511 \mathrm{Mb}$ with 27 pseudochromosomes. A comparative genomic study indicated that $P$. patens shared a high degree of homology with high plants such as A. thaliana (Reski et al., 1998; Nishiyama et al., 2003). For example, more than $66 \%$ of $A$. thaliana proteins have homologs in $P$. patens. When combined with other advantages such as a completely sequenced genome, short life cycle, easy culture and maintenance, simple morphology, and polarized tip growing rhizoids and protonema, Physcomitrella patens becomes a powerful model system for the study of gene functions (Cove, 2005; Quatrano et al., 2007; Prigge and Bezanilla, 2010; Vidali and Bezanilla, 2012; Jaeger and Moody, 2021). More importantly, the predominant phase in the $P$. patens life cycle, from the germination of spores to the fertilization of eggs, is haploid, which allows screening of knockout mutant could complete in one generation.

\section{GENERATION OF SUCCESSFUL GENE KNOCKOUTS BY GENE TARGETING}

\section{Design of Knockout Constructs}

To generate targeted knockouts efficiently, a good design of knockout cassettes is necessary. Typically, a knockout construct containing a selection marker flanked by left and right homologous arms comprising either cDNA or genomic sequences homologous to the target sites. Hygromycin or Geneticin resistant genes are common selection markers in the knockout cassettes (Schaefer and Zryd, 1997). Selection markers should insert into exons to ensure their expression in the regenerated plants. PHYSCObase provides a preassembled plasmid pTN80 (Accession: AB267704.2, G.I: $379,990,978)$ to the community to easily construct a knockout cassette. Figure 1A illustrates a knockout cassette prepared from pTN80, which comprises the $n p t$ ii gene as a selection marker driven by a $35 \mathrm{~S}$ promoter and tailed with a nos terminator. Homologous arm sequences with equivalent length could target conserved domains, activation sites, promoters, or UTRs. $1 \mathrm{~kb}$ homologous sequences are sufficient to obtain gene knockouts, although 600-700 bp long homologous arms are also adequate to generate successful knockout transformants (Kamisugi et al., 2005). The length of homologous arms is correlated to the ontarget insertions (Shy et al., 2016). 


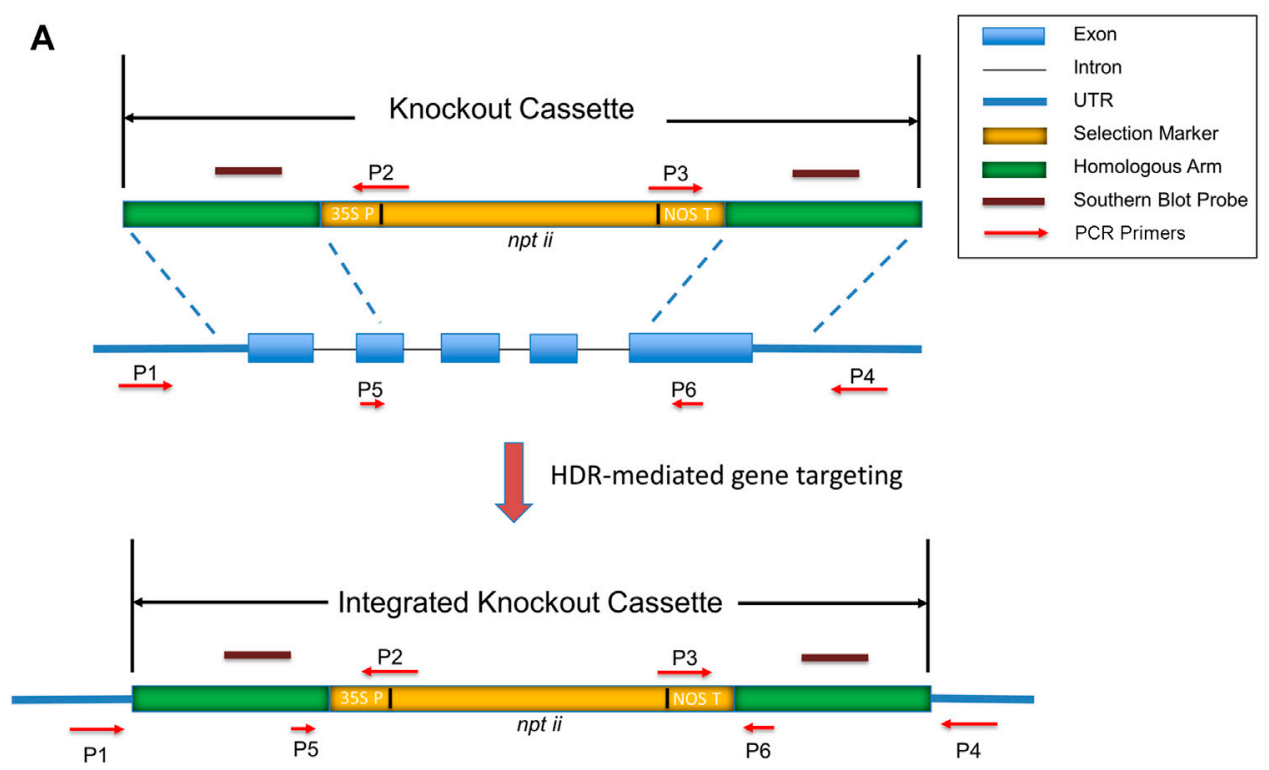

B

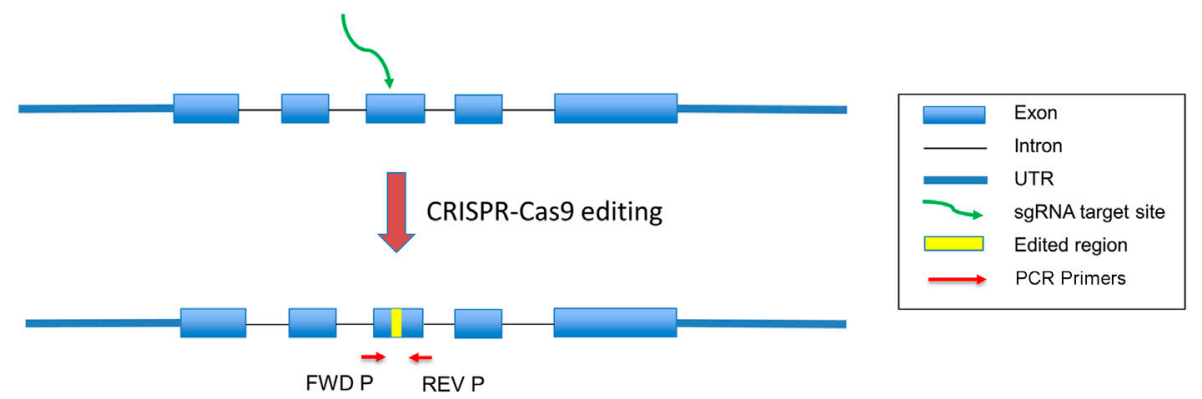

FIGURE 1 | Targeted gene knockout generated by gene targeting and CRISPR-Cas9. (A) A successful gene targeting event results in targeted gene replacement. The knockout cassette contains homologous arms flanking npt ii genes driven by 35S promoter and tailed with a nos terminator. By transformation of a linearized knockout cassette to the moss protoplasts, the marker gene nptii is integrated into the genome, resulting in targeted gene replacement. (B) A frameshift mutant generated by CRISPR-Cas9. The yellow bar indicates gene-edited sequences. The green arrow suggests the target site. Red arrows show the location of primers (FWD P and REV P) for the amplification of the edited site.

\section{Protoplast Transformation and Regeneration}

Two decades ago, when gene targeting emerged, particle bombardment transformation to vegetative tissue or PEGmediated transformation to protoplasts were both used by researchers (Cho et al., 1999; Bezanilla et al., 2003). Recently, the transformation of knockout cassettes to protoplasts has become prevalent in Physcomitrella gene targeting (Schaefer et al., 1991; Schaefer and Zryd, 1997; Rensing et al., 2020). PEG-mediated transformation and protoplast regeneration protocols are available on PHYSCObase and CSH protocol (Cove et al., 2009a). Moss protoplasts are filamentous protonema of which cell walls are degraded by Drislease (Grimsley et al., 1977). Both circular and linearized knockout constructs can use in the transformation. However, circular DNA may undergo extrachromosomal replication in transient knockouts and reduce the number of stable transformants. Therefore, linearized knockout cassettes are preferably transformed into protoplasts (Strepp et al., 1998; Ashton et al., 2000; Mittmann et al., 2004). Transformed protoplasts resuspended in PRMB medium incubate in the dark overnight and regenerate for 7 days under normal growth conditions. The two-round selection of stable transformants is carried out on the solid culture medium containing antibiotics for 2-3 weeks per round with a one- or 2-week interval on an antibiotic-free medium.

\section{Challenges and Technical Difficulties}

Even though targeted gene knockouts mediated by homologous recombination are potent tools, there are challenges and fundamental issues with gene targeting. First, off-target events are the major challenge in gene targeting. The donor knockout cassettes transformed to the protoplasts integrate into the moss 
TABLE 1 | Comparison of the use of gene targeting and CRISPR-Cas9 in Physcomitrella patens. Discussion topics cover constructs, protoplast transformation and regeneration, selection of knockouts, molecular analysis, mutant types, efficiency, and multiplexing knockouts.

\begin{tabular}{|c|c|c|c|}
\hline & CRISPR-Cas9 Technology & Gene Targeting & References \\
\hline Construct & sgRNA and Cas9 plasmids & Knockout cassette & $\begin{array}{l}\text { Collonnier et al. (2017) } \\
\text { Mallett et al. (2019) } \\
\text { Girke et al. (1998) }\end{array}$ \\
\hline $\begin{array}{l}\text { Protoplast Transformation and } \\
\text { Regeneration }\end{array}$ & PEG-mediated protoplast transformation & PEG-mediated protoplast transformation & $\begin{array}{l}\text { Schaefer \& Zryd (1997) } \\
\text { Cove et al. (2009a) } \\
\text { Radin et al. (2021) }\end{array}$ \\
\hline Selection of Knockouts & $\begin{array}{l}\text { Selection of regenerated plants harboring } \\
\text { transiently expressed Cas } 9 \text { and sgRNA; } \\
\text { 1-week selection on antibiotics medium }\end{array}$ & $\begin{array}{l}\text { Selection of regenerated plants comprising stable } \\
\text { integration of knockout cassettes; } \\
\text { Two rounds of selection on antibiotic medium with a } \\
\text { 1-week interval }\end{array}$ & $\begin{array}{l}\text { Cove et al. (2009b) } \\
\text { Hiwatashi \& Hasebe (2012) }\end{array}$ \\
\hline Molecular Analysis & PCR, T7 endonuclease assay, & PCR, RT-PCR, Southern blot & $\begin{array}{l}\text { Steinberger et al. (2021) } \\
\text { Gomann et al. (2021) } \\
\text { Mallett et al. (2019) } \\
\text { Khandelwal et al. (2010) }\end{array}$ \\
\hline Mutant Types & $\begin{array}{l}\text { Frameshift mutants (knockouts), } \\
\text { Base-edited mutants, } \\
\text { Marker-free mutants, } \\
\text { Loss-of-function or gain-of-function allele }\end{array}$ & $\begin{array}{l}\text { Gene knockouts, } \\
\text { Knock-in mutant, } \\
\text { Marker gene or tag integrated to moss genome, } \\
\text { Complementation line }\end{array}$ & $\begin{array}{l}\text { Guyon-Debast et al. (2021) } \\
\text { Radin et al. (2021); } \\
\text { Brejskova et al. (2021) } \\
\text { Schaefer (2001) }\end{array}$ \\
\hline $\begin{array}{l}\text { Efficiency (targeted mutant/ } \\
\text { regenerated protoplasts) }\end{array}$ & $2-3 \%$ & $0.25 \%$ & Collonnier et al. (2017) \\
\hline Multiplexing Knockouts & Simple, by the single transformation event & $\begin{array}{l}\text { Time-consuming, usually by sequential } \\
\text { transformations }\end{array}$ & $\begin{array}{l}\text { Trogu et al. (2020) } \\
\text { Lopez-Obando et al. (2016) } \\
\text { Takechi et al. (2021) }\end{array}$ \\
\hline
\end{tabular}

genome by either targeted gene replacement, targeted gene insertion, or illegitimate insertion (Kamisugi et al., 2005; Kamisugi et al., 2006). Among the three integrations, targeted gene replacement is ideal for integrating two homologous arms to the target loci by high-frequency homologous recombination in Physcomitrella patens. However, targeted insertions with one end on-target while the other end off-target is commonly present. Meanwhile, insertions of knockout cassettes randomly into the moss genome mediated by NHEJ might associate with targeted gene replacement. Additionally, researchers have found that multiple linearized knockout cassettes might form concatemers during the protoplast transformation (Kamisugi et al., 2006).

Validation of knockouts containing a single targeted gene replacement event is therefore essential before the phenotypic analysis. PCR is the primary method to confirm a single-copy integration of the exogenous knockout construct into the moss genome. As shown in Figure 1A, primer pairs P1/P2 and P3/P4 can amplify the insertion junctions of $5^{\prime}$ and $3^{\prime}$ ends, respectively. P5 and P6 are a pair of primers for the detection of RNA transcription to confirm the depletion of the targeted region. Southern blot furtherly confirms the single-copy insertion and excludes the ectopic insertions on the moss genome. The probes hybridize to the two homologous arms (Khandelwal et al., 2010).

Secondly, Protoplast fusion is present in the PEG-mediated transformation (Grimsley et al., 1977). The two broken protoplasts may fuse to make a diploid cell with one copy of the knockout allele and another copy of the wild-type allele during transformation and regeneration. For this case, PCR primers P1/P4 illustrated in Figure $\mathbf{1 A}$ can identify the wildtype gene copy in the knockouts.

Thirdly, the knockout of essential genes and multiple-gene families is challenging. Depletion of crucial genes often results in lethal mutants. Knockout multiple gene families or genes with redundancy are also tricky with gene targeting. Transformation of multiple knockouts constructs simultaneously to protoplasts may result in concatemers in the target loci.

\section{CRISPR-CAS9 MEDIATED GENOME EDITING}

CRISPR-Cas9 system as an efficient genome editing tool, in which Cas9 cleaves DNA at target sites specified by guide RNA, has been applied to many plant species, including Physcomitrella patens. (Li et al., 2012; Shan et al., 2013; Rensing et al., 2020; Shan et al., 2020). Repair pathways of double-strand breaks (DSBs) in the traditional gene targeting and the CRISPR-Cas9 system are different. Gene targeting depends on homologous recombination, whereas Cas9induced breaks are mainly repaired by NHEJ or alternative endjoining (Alt-EJ) (Collonnier et al., 2017). CRISPR/Cas9 can produce deletions, insertions, and substitutions, which could be frameshift mutants, early terminations, splicing variants, etc. Figure $\mathbf{1 B}$ shows a targeting site edited by CRISPR-Cas 9 system.

To compare CRISPR-Cas9 and traditional gene targeting in detail, Table 1 lists aspects related to using two technologies in Physcomitrella patens, covering topics from vector construct, 
protoplast transformation and regeneration, selection of mutants, molecular analysis to mutant types, multiplexing options, and efficiency. Additionally, scientists have expanded the CRISPRCas9 toolkit to control gene editing tightly.

\section{Construct of sgRNA and Cas9}

Compared to the knockout cassette used in gene targeting, Cas9 and sgRNA are two components required for delivery to host cells in the CRISPR-Cas9 system (Collonnier et al., 2017; Mallett et al., 2019). Importantly, guide RNA specificity is critical for efficient editing through CRISPR-Cas9. To minimize the off-target events, specific gDNA sequences can be obtained in software called CRISPOR (Haeussler et al., 2016). Synthesized gDNA (sgDNA) and Cas9 are cloned to plasmids harboring additional selection marker genes that assist the transient selection of the regenerated protoplasts (Trogu et al., 2020; Yi and Goshima, 2020).

Promoters for the expression of Cas 9 and sgRNA are studied. The most commonly used promoters include rice actin (Act) promoter and maize ubiquitin promoter for the expression of Cas9, and Physcomitrella patens U6 promoter for driving the expression of sgRNAs (Mallett et al., 2019). SgRNA and Cas9 plasmid should be at an equal ratio when transformed to protoplasts (Collonnier et al., 2017). Instead of separate plasmids for Cas9 and sgRNA, a modular CRISPR-Cas9 vector system has been developed to drive the expression of Cas9 and multiple sgRNAs simultaneously (Mallett et al., 2019). A successful application of this vector system was to generate seven mutant lines targeting the $\mathrm{SBH}$ gene (Steinberger et al., 2021). In the same vector system, up to 4 sgRNAs can be assembled with three choices of antibiotics resistant genes, hygromycin, G418, and zeocin, which enable targeting up to 12 genome sites in a single transformation.

\section{Protoplast Transformation and Regeneration}

Delivery of Cas9 and sgRNA to plants could be achieved by Agrobacterium-mediated stable transformation. The transformation rate for protoplast transformation using Agrobacterium is typically $10^{-4}$, calculated by the number of stable transformants divided by surviving regenerants after transformation (Cove et al., 2009b). However, Agrobacterium-mediated transformation involves in the integration of exogenous DNA into target plants and the resulting mutants could be considered as genetically modified organisms. Instead, the protoplast transformation of plasmids that transiently express in plant cells will generate transgene-free knockout mutants. Although protoplast transformation and regeneration are a bottleneck and are currently developing in many plant species (Hsu et al., 2021; Li et al., 2021), PEG-mediated protoplast transformation has been widely used in P. patens study for gene targeting, including steps of cell wall degradation, protoplast resuspension, plasmid transformation, and protoplast regeneration and selection of knockouts on growth medium (Schaefer et al., 1991; Schaefer, 2001; Cove et al., 2009a). The same protocol can be utilized in the CRISPR-Cas9 system, except for different vectors for transformation (Lopez-Obando et al., 2016; Collonnier et al., 2017; Radin et al., 2021). 10-30 ug of Cas9 and sgRNA constructs are introduced to the $4.8 \times 10^{5}$ resuspended protoplasts. Co-transformation of transiently expressed marker genes with Cas9 and sgRNA plasmids enables the reduction of false-positive clones (Gomann et al., 2021). Upon transformation, protoplasts grow on the cellophane overlaid on the regeneration medium for 4-7 days (Cove et al., 2009b; Radin et al., 2021).

\section{Selection of Knockouts}

Knockout selection in CRISPR-Cas9 system can be completed in a week. Compared to the 1-month selection of stable knockouts by gene targeting, selection of transiently expressed Cas 9 and sgRNA only takes 7 days. One round of selection against proper antibiotics is sufficient to confirm the Cas9 and sgRNA presence in the regenerated clones. This saves time to generate knockouts and increases the number of regenerated plants, as shown in a more significant number of clones emerging from the first-round selection than that from the 2 nd round selection on the medium conferring antibiotic resistance (Hiwatashi and Hasebe, 2012).

\section{Molecular Analysis of Putative Knockouts}

Whether in traditional gene targeting or CRISPR-Cas9 system, individual clones are sub-cultured on a standard growth medium for 2-3 weeks after selection. Protonema or young gametophytes are harvested to extract gDNA for molecular analysis of mutants. The transient expression of Cas9 and sgRNA in CRIPSR-Cas9 system allows for an increased number of regenerated plants. However, this requires an expanding screening work to identify edited plants from non-transgenic and non-edited surviving plants after transformation and regeneration.

For knockout screening, PCR amplification around the expected editing sites is a primary method to validate knockouts generated by gene targeting and CRISPR-Cas9. Additionally, unlike analysis methods such as RT-PCR and Southern blotting used in gene targeting, T7 endonuclease assay is utilized to screen potential knockouts in the CRISPRCas9 technique (Mallett et al., 2019). Screening of a large number of mutants can be performed by visualizing amplicons on 3\% of agarose gel or the high-resolution PAGE gel (LopezObando et al., 2016; Trogu et al., 2020). In this way, clones with base-pair changes can be detected on the gel and subsequently sequenced. Off-target events in CRISPR-Cas9 are also checked by amplifying potential off-target sites, but often no mutations on these putative off-target loci are detected (Collonnier et al., 2017).

\section{Mutant Types}

Successful gene targeting events can generate both targeted gene knockouts and knock-in alleles at expected genomic sites. Those knockout or knock-in lines comprise selection markers or tags stably integrated into the genome (Schaefer, 2001). Furthermore, gene targeting can produce complementation lines to rescue the mutant phenotype by targeting full-length cDNA to the moss genome (Brejskova et al., 2021).

Knockouts generated by typical CRISPR-Cas9 resulted from NHEJ or Alt-NJ, harbor deletions, insertions, and rarely substitutions, most of which are frameshift mutations (LopezObando et al., 2016; Mallett et al., 2019). Furthermore, in combination with donor DNA templates, CRISPR-Cas9 can also generate knock-in and gain-of-function alleles (Guyon-Debast et al., 
2021; Radin et al., 2021). We will discuss this in the later section. Overall, CRISPR-Cas9 edited knockouts are free of the transgene.

\section{Knockout Efficiency}

Traditional gene targeting is only limited to certain organisms such as mice, yeast, etc. Although the homologous recombination rate is much high in $P$. patens, obtaining successful knockout lines through homologous recombination is not as highly efficient as the CRISPRCas9 system. As shown in the literature, the traditional gene targeting only resulted in $0.25 \%$ relative transformation efficiency, whereas CRISPR-Cas9 reached 2.1-3.2\% relative transformation efficiency from different sgRNAs using $P p A P T$ as a target gene (Collonnier et al., 2017). That being said, for 15,000 regenerated clones, about $315 \sim 480$ knockouts survived in the CRISPR-Cas9 editing system, but only 37 knockouts emerged from traditional gene targeting.

\section{Multiplexing Knockouts}

Physcomitrella patens have undergone genome duplications, and thus many gene families expanded to contain multiple genes (Zimmer et al., 2013; Li et al., 2015). Therefore, generation knockouts of multiple genes are necessary to study gene family functions. Both traditional gene targeting and the CRISPR-Cas9 system can generate multi-gene knockouts. However, multi-gene targeting using traditional gene targeting is time-consuming, associated with many rounds of selection (Takechi et al., 2021). In comparison, the generation of multi-gene knockouts using CRISPR-Cas9 is much easier and simpler. Co-transformation of Cas9 and multiple sgRNAs could result in multiplex gene knockouts. For example, a septuple knockout mutant was generated by a single transformation of Cas 9 and sgRNAs (Trogu et al., 2020). Multiple genes from different gene families can also be targeted in the CRISPR-Cas9 system. Notably, single or double mutants may be present as byproducts during the transformation of multiple sgRNAs and Cas9 to moss protoplasts, creating genetic variations (Lopez-Obando et al., 2016).

\section{Editing Physcomitrella patens Genome Precisely and Efficiently}

CRISPR-Cas9 is an efficient gene-editing system that results in deletions, insertions, and substitutions. Because Physcomitrella patens has a high frequency of homologous recombination, scientists aim to take advantage of this feature to control CRISPR-Cas9 editing tightly in the moss. Donor DNA templates are co-transformed with plasmids harboring Cas9 and sgRNAs to moss protoplasts. Collonnier reported that $60 \%$ of CRISPR-Cas9 induced DSBs were repaired via HDR, compared to $54 \%$ gene targeting efficiency shown in the same paper (Collonneir et al., 2017). Either single-strand (ss) DNA or double-strand (ds) DNA oligos, linearized plasmids, or circular plasmids can function as donor DNA templates (Collonneir et al.,

\section{REFERENCES}

Ashton, N. W., Champagne, C. E. M., Weiler, T., and Verkoczy, L. K. (2000). The Bryophyte Physcomitrella Patens Replicates Extrachromosomal Transgenic Elements. New Phytol. 146, 391-402. doi:10.1046/j.1469-8137.2000.00671.x
2017; Mallett et al., 2019; Yi and Goshima, 2020; Radin et al., 2021). Consequently, DSB repair is tightly controlled by HDR, and genome editing in $P$. patens becomes more precise, resulting in $28-100 \%$ of colonies showing expected gene editing including substitutions, deletions, and knock-in tagging at target sites (Yi and Goshima, 2020). The donor DNA template-assisted or called oligodeoxynucleotide (ODN)-assisted CRISPR-Cas9 method generates knockouts, knock-in lines, and substitutions, which could be loss-of-function alleles, gain-of-function alleles, or hypoalleles that are beneficial for the study of essential genes.

Furtherly, a more precise mutation system called CRISPRmediated base editors (BEs) has been developed in human murine cell lines, rice, and as well as in Physcomitrella patens (Komor et al., 2016; Li et al., 2017; Lu and Zhu, 2017; Guyon-Debast et al., 2021). In $P$. patens, CRISPR-Cas9 deaminase systems CBE was designed to obtain cytosine editing and $\mathrm{ABE}$ system was aimed for adenine editing in an predictable editing window, which was about -20 to $-14 \mathrm{bp}$ from PAM sites (Guyon-Debast et al., 2021). By co-transformation of sgRNAs specific to the reporter PpAPT gene driven by snRNA U6 promoter and nCas9 (D10A) fused with either Petromyzon marinus cytosine deaminase driven by pcUbi4-2 promoter or a heterodimer of wild-type and mutated E. coli tRNA adenosine deaminase driven by OsAct1 promoter, $89 \%$ mutants from a 2-FA selection corresponded to precise base editing through CBE system and $100 \%$ mutants survived on a 2-FA selection corresponded to A-to-G base editing in ABE system. For other targeting genes, $\mathrm{CBE}$ could result in up to $55 \%$ efficiency. The same study also showed that multiplex (up to 4 sgRNA targets) base editing was possible in Physcomitrella patens with $\mathrm{CBE}$ and $\mathrm{ABE}$ strategies.

\section{CONCLUSIONS}

The moss Physcomitrella patens is a unique model organism harboring a high frequency of homologous recombination. Gene targeting has been widely used in Physcomitrella patens for the generation of targeted gene replacement. Recently, CRISPR-Cas9 technology is a hot topic in the genome-editing field. With its high efficiency and the resulting marker-free knockouts, CRISPRCas9 becomes increasingly appealing to scientists who work on Physcomitrella patens.

\section{AUTHORS CONTRIBUTIONS}

LZ wrote the manuscript.

\section{ACKNOWLEDGMENTS}

Thanks to both editor and reviewers for reviewing, editorial comments, and suggestions for improvement of the manuscript.

Bezanilla, M., Pan, A., and Quatrano, R. S. (2003). RNA Interference in the Moss Physcomitrella Patens. Plant Physiol. 133 (2), 470-474. doi:10.1104/pp.103.024901 Bouabe, H., and Okkenhaug, K. (2013). Gene Targeting in Mice: a Review. Methods Mol. Biol. 1064, 315-336. doi:10.1007/978-1-62703-601-6_23

Brejšková, L., Hála, M., Rawat, A., Soukupová, H., Cvrčková, F., Charlot, F., et al. (2021). SEC6 Exocyst Subunit Contributes to Multiple Steps of Growth and 
Development of Physcomitrella (Physcomitrium Patens). Plant J. 106 (3), 831-843. doi:10.1111/tpj.15205

Capecchi, M. (2005a). An Interview with the 2007 Nobel Laureates in Physiology or Medicine. Nat. Rev. Genet. 6, 434. doi:10.1038/nrg1647

Capecchi, M. R. (2005b). Gene Targeting in Mice: Functional Analysis of the Mammalian Genome for the Twenty-First century. Nat. Rev. Genet. 6, 507-512. doi: $10.1038 / \mathrm{nrg} 1619$

Cho, S. H., Chung, Y. S., Cho, S. K., Rim, Y. W., and Shin, J. S. (1999). Particle Bombardment Mediated Transformation and GFP Expression in the moss Physcomitrella Patens. Mol. Cell 9 (1), 14-19. Available From: https://www. ncbi.nlm.nih.gov/pubmed/10102565.

Collonnier, C., Epert, A., Mara, K., Maclot, F., Guyon-Debast, A., Charlot, F., et al. (2017). CRISPR-Cas9-mediated Efficient Directed Mutagenesis and RAD51dependent and RAD51-independent Gene Targeting in the mossPhyscomitrella Patens. Plant Biotechnol. J. 15 (1), 122-131. doi:10.1111/pbi.12596

Cong, L., Ran, F. A., Cox, D., Lin, S., Barretto, R., Habib, N., et al. (2013). Multiplex Genome Engineering Using CRISPR/Cas Systems. Science 339 (6121), 819-823. doi:10.1126/science. 1231143

Cove, D. J., Perroud, P.-F., Charron, A. J., McDaniel, S. F., Khandelwal, A., and Quatrano, R. S. (2009a). The moss Physcomitrella Patens: a Novel Model System for Plant Development and Genomic Studies. Cold Spring Harb Protoc. 2009 (2), pdb.emo115. doi:10.1101/pdb.emo115

Cove, D. J., Perroud, P.-F., Charron, A. J., McDaniel, S. F., Khandelwal, A., and Quatrano, R. S. (2009b). Transformation of the moss Physcomitrella Patens Using T-DNA Mutagenesis. Cold Spring Harb Protoc. 2009 (2), pdb.prot5144. doi:10.1101/pdb.prot5144

Cove, D. (2005). The moss Physcomitrella Patens. Annu. Rev. Genet. 39, 339-358. doi:10.1146/annurev.genet.39.073003.110214

Engel, P. P. (1968). The Induction of Biochemical and Morphological Mutants in the moss Physcomitrella Patens. Am. J. Bot. 55, 438-446. doi:10.1002/j.15372197.1968.tb07397.x

Ermert, A. L., Nogué, F., Stahl, F., Gans, T., and Hughes, J. (2019). CRISPR/Cas9Mediated Knockout of Physcomitrella Patens Phytochromes. Methods Mol. Biol. 2026, 237-263. doi:10.1007/978-1-4939-9612-4_20

Gardner, J. M., and Jaspersen, S. L. (2014). Manipulating the Yeast Genome: Deletion, Mutation, and Tagging by PCR. Methods Mol. Biol. 1205, 45-78. doi:10.1007/978-1-4939-1363-3_5

Gerlai, R. (2016). Gene Targeting Using Homologous Recombination in Embryonic Stem Cells: The Future for Behavior Genetics?. Front. Genet. 7, 43. doi:10.3389/fgene.2016.00043

Girke, T., Schmidt, H., Zahringer, U., Reski, R., and Heinz, E. (1998). Identification of a Novel D6-Acyl-Group Desaturase by Targeted Gene Disruption in Physcomitrella Patens. Plant J. 15 (1), 39-48. doi:10.1046/j.1365-313x.1998.00178.x

Gömann, J., Herrfurth, C., Zienkiewicz, K., Haslam, T. M., and Feussner, I. (2021). Sphingolipid $\Delta 4$-desaturation Is an Important Metabolic Step for Glycosylceramide Formation in Physcomitrium Patens. J. Exp. Bot. 72 (15), 5569-5583. doi:10.1093/jxb/erab238

Grimsley, N. H., Ashton, N. W., and Cove, D. J. (1977). The Production of Somatic Hybrids by Protoplast Fusion in the moss, Physcomitrella Patens. Mol. Gen. Genet. 154, 97-100. doi:10.1007/bf00265582

Guyon-Debast, A., Alboresi, A., Terret, Z., Charlot, F., Berthier, F., Vendrell-Mir, P., et al. (2021). A Blueprint for Gene Function Analysis through Base Editing in the Model Plant Physcomitrium (Physcomitrella) Patens. New Phytol. 230 (3), 1258-1272. doi:10.1111/nph.17171

Guyon-Debast, A., Rossetti, P., Charlot, F., Epert, A., Neuhaus, J.-M., Schaefer, D. G., et al. (2019). The XPF-ERCC1 Complex Is Essential for Genome Stability and Is Involved in the Mechanism of Gene Targeting in Physcomitrella Patens. Front. Plant Sci. 10, 588. doi:10.3389/fpls.2019.00588

Haeussler, M., Schönig, K., Eckert, H., Eschstruth, A., Mianné, J., Renaud, J.-B., et al. (2016). Evaluation of Off-Target and On-Target Scoring Algorithms and Integration into the Guide RNA Selection Tool CRISPOR. Genome Biol. 17 (1), 148. doi:10.1186/s13059-016-1012-2

Hiwatashi, Y., and Hasebe, M. (2012). How to Transform Physcomitrella Patens: PEG-Mediatedtransformation. http://moss.nibb.ac.jp/.

Hsu, C.-T., Lee, W.-C., Cheng, Y.-J., Yuan, Y.-H., Wu, F.-H., and Lin, C.-S. (2021). Genome Editing and Protoplast Regeneration to Study Plant-Pathogen Interactions in the Model Plant Nicotiana Benthamiana. Front. Genome Ed. 2, 627803. doi:10.3389/fgeed.2020.627803
Hsu, P. D., Lander, E. S., and Zhang, F. (2014). Development and Applications of CRISPR-Cas9 for Genome Engineering. Cell 157 (6), 1262-1278. doi:10.1016/ j.cell.2014.05.010

Jaeger, R., and Moody, L. A. (2021). A Fundamental Developmental Transition inPhyscomitrium Patensis Regulated by Evolutionarily Conserved Mechanisms. Evol. Dev. 23 (3), 123-136. doi:10.1111/ede.12376

Jiang, W., Zhou, H., Bi, H., Fromm, M., Yang, B., and Weeks, D. P. (2013). Demonstration of CRISPR/Cas9/sgRNA-mediated Targeted Gene Modification in Arabidopsis, Tobacco, Sorghum and rice. Nucleic Acids Res. 41 (20), e188. doi:10.1093/nar/gkt780

Kamisugi, Y., Cuming, A. C., and Cove, D. J. (2005). Parameters Determining the Efficiency of Gene Targeting in the moss Physcomitrella Patens. Nucleic Acids Res. 33 (19), e173. doi:10.1093/nar/gni172

Kamisugi, Y., Schaefer, D. G., Kozak, J., Charlot, F., Vrielynck, N., Holá, M., et al. (2012). MRE11 and RAD50, but Not NBS1, Are Essential for Gene Targeting in the moss Physcomitrella Patens. Nucleic Acids Res. 40 (8), 3496-3510. doi:10.1093/nar/gkr1272

Kamisugi, Y., Schlink, K., Rensing, S. A., Schween, G., von Stackelberg, M., Cuming, A. C., et al. (2006). The Mechanism of Gene Targeting in Physcomitrella Patens: Homologous Recombination, Concatenation and Multiple Integration. Nucleic Acids Res. 34 (21), 6205-6214. doi:10.1093/ nar/gkl832

Kamisugi, Y., Whitaker, J. W., and Cuming, A. C. (2016). The Transcriptional Response to DNA-Double-Strand Breaks in Physcomitrella Patens. PLoS One 11 (8), e0161204. doi:10.1371/journal.pone.0161204

Khandelwal, A., Cho, S. H., Marella, H., Sakata, Y., Perroud, P.-F., Pan, A., et al. (2010). Role of ABA and ABI3 in Desiccation Tolerance. Science 327 (5965), 546. doi:10.1126/science.1183672

Knott, G. J., and Doudna, J. A. (2018). CRISPR-cas Guides the Future of Genetic Engineering. Science 361 (6405), 866-869. doi:10.1126/science.aat5011

Komor, A. C., Kim, Y. B., Packer, M. S., Zuris, J. A., and Liu, D. R. (2016). Programmable Editing of a Target Base in Genomic DNA without DoubleStranded DNA Cleavage. Nature 533 (7603), 420-424. doi:10.1038/ nature17946

Li, F.-W., Melkonian, M., Rothfels, C. J., Villarreal, J. C., Stevenson, D. W., Graham, S. W., et al. (2015). Phytochrome Diversity in green Plants and the Origin of Canonical Plant Phytochromes. Nat. Commun. 6, 7852. doi:10.1038/ ncomms 8852

Li, J., Sun, Y., Du, J., Zhao, Y., and Xia, L. (2017). Generation of Targeted Point Mutations in Rice by a Modified CRISPR/Cas9 System. Mol. Plant 10 (3), 526-529. doi:10.1016/j.molp.2016.12.001

Li, T., Liu, B., Spalding, M. H., Weeks, D. P., and Yang, B. (2012). High-efficiency TALEN-Based Gene Editing Produces Disease-Resistant rice. Nat. Biotechnol. 30 (5), 390-392. doi:10.1038/nbt.2199

Li, X., Sandgrind, S., Moss, O., Guan, R., Ivarson, E., Wang, E. S., et al. (2021). Efficient Protoplast Regeneration Protocol and CRISPR/Cas9-Mediated Editing of Glucosinolate Transporter (GTR) Genes in Rapeseed (Brassica Napus L.). Front. Plant Sci. 12, 680859. doi:10.3389/fpls.2021.680859

Liang, Z., Zhang, K., Chen, K., and Gao, C. (2014). Targeted Mutagenesis in Zea mays Using TALENs and the CRISPR/Cas System. J. Genet. Genomics 41 (2), 63-68. doi:10.1016/j.jgg.2013.12.001

Lopez-Obando, M., Hoffmann, B., Géry, C., Guyon-Debast, A., Téoulé, E., Rameau, C., et al. (2016). Simple and Efficient Targeting of Multiple Genes through CRISPR-Cas9 in Physcomitrella Patens. G3 (Bethesda) 6 (11), 3647-3653. doi:10.1534/g3.116.033266

Lu, Y., and Zhu, J.-K. (2017). Precise Editing of a Target Base in the Rice Genome Using a Modified CRISPR/Cas9 System. Mol. Plant 10 (3), 523-525. doi:10.1016/j.molp.2016.11.013

Mallett, D. R., Chang, M., Cheng, X., and Bezanilla, M. (2019). Efficient and Modular CRISPR-Cas9 Vector System for Physcomitrella Patens. Plant Direct 3 (9), e00168. doi:10.1002/pld3.168

Markmann-Mulisch, U., Wendeler, E., Zobell, O., Schween, G., Steinbiss, H.-H., and Reiss, B. (2007). Differential Requirements for RAD51 inPhyscomitrella patensandArabidopsis thalianaDevelopment and DNA Damage Repair. Plant Cell 19 (10), 3080-3089. doi:10.1105/tpc.107.054049

Miller, J. C., Holmes, M. C., Wang, J., Guschin, D. Y., Lee, Y.-L., Rupniewski, I., et al. (2007). An Improved Zinc-finger Nuclease Architecture for Highly Specific Genome Editing. Nat. Biotechnol. 25 (7), 778-785. doi:10.1038/nbt1319 
Mittmann, F., Brucker, G., Zeidler, M., Repp, A., Abts, T., Hartmann, E., et al. (2004). Targeted Knockout in Physcomitrella Reveals Direct Actions of Phytochrome in the Cytoplasm. Proc. Natl. Acad. Sci. 101 (38), 13939-13944. doi:10.1073/pnas.0403140101

Nekrasov, V., Staskawicz, B., Weigel, D., Jones, J. D. G., and Kamoun, S. (2013). Targeted Mutagenesis in the Model Plant Nicotiana Benthamiana Using Cas9 RNAGuided Endonuclease. Nat. Biotechnol. 31 (8), 691-693. doi:10.1038/nbt.2655

Nishiyama, T., Fujita, T., Shin-I, T., Seki, M., Nishide, H., Uchiyama, I., et al. (2003). Comparative Genomics of Physcomitrella Patens Gametophytic Transcriptome and Arabidopsis thaliana: Implication for Land Plant Evolution. Proc. Natl. Acad. Sci. 100 (13), 8007-8012. doi:10.1073/pnas.0932694100

Prigge, M. J., and Bezanilla, M. (2010). Evolutionary Crossroads in Developmental Biology: Physcomitrella Patens. Development 137 (21), 3535-3543. doi:10.1242/ dev.049023

Quatrano, R., McDaniel, S., Khandelwal, A., Perroud, P., and Cove, D. (2007). Physcomitrella Patens: Mosses Enter the Genomic Age. Curr. Opin. Plant Biol. 10 (2), 182-189. doi:10.1016/j.pbi.2007.01.005

Radin, I., Richardson, R. A., Coomey, J. H., Weiner, E. R., Bascom, C. S., Li, T., et al. (2021). Plant PIEZO Homologs Modulate Vacuole Morphology during Tip Growth. Science 373 (6554), 586-590. doi:10.1126/science.abe6310

Ran, F. A., Hsu, P. D., Wright, J., Agarwala, V., Scott, D. A., and Zhang, F. (2013). Genome Engineering Using the CRISPR-Cas9 System. Nat. Protoc. 8 (11), 2281-2308. doi:10.1038/nprot.2013.143

Rensing, S. A., Goffinet, B., Meyberg, R., Wu, S.-Z., and Bezanilla, M. (2020). The Moss Physcomitrium (Physcomitrella) Patens: A Model Organism for Nonseed Plants. Plant Cell 32 (5), 1361-1376. doi:10.1105/tpc.19.00828

Rensing, S. A., Lang, D., Zimmer, A. D., Terry, A., Salamov, A., Shapiro, H., et al. (2008). The Physcomitrella Genome Reveals Evolutionary Insights into the Conquest of Land by Plants. Science 319 (5859), 64-69. doi:10.1126/ science.1150646

Reski, R., Reynolds, S., Wehe, M., Kleber-Janke, T., and Kruse, S. (1998). Moss (Physcomitrella Patens) Expressed Sequence Tags Include Several Sequences Which Are Novel for Plants. Bot. Acta 111, 6. doi:10.1111/j.14388677.1998.tb00689.x

Sander, J. D., Dahlborg, E. J., Goodwin, M. J., Cade, L., Zhang, F., Cifuentes, D., et al. (2011). Selection-free zinc-finger-nuclease Engineering by Context-dependent Assembly (CoDA). Nat. Methods 8 (1), 67-69. doi:10.1038/nmeth.1542

Schaefer, D. G., Delacote, F., Charlot, F., Vrielynck, N., Guyon-Debast, A., Le Guin, S., et al. (2010). RAD51 Loss of Function Abolishes Gene Targeting and Derepresses Illegitimate Integration in the moss Physcomitrella Patens. DNA Repair 9 (5), 526-533. doi:10.1016/j.dnarep.2010.02.001

Schaefer, D. (2001). Gene Targeting in Physcomitrella Patens. Curr. Opin. Plant Biol. 4 (2), 143-150. doi:10.1016/s1369-5266(00)00150-3

Schaefer, D. G., and Zryd, J.-P. (1997). Efficient Gene Targeting in the moss Physcomitrella Patens. Plant J. 11 (6), 1195-1206. doi:10.1046/j.1365313x.1997.11061195.xAvailable From: https://www.ncbi.nlm.nih.gov/ pubmed/9225463

Schaefer, D., Zryd, J.-P., Knight, C. D., and Cove, D. J. (1991). Stable Transformation of the moss Physcomitrella Patens. Mol. Gen. Genet. 226 (3), 418-424. doi:10.1007/BF00260654

Shan, Q., Wang, Y., Li, J., Zhang, Y., Chen, K., Liang, Z., et al. (2013). Targeted Genome Modification of Crop Plants Using a CRISPR-Cas System. Nat. Biotechnol. 31 (8), 686-688. doi:10.1038/nbt.2650

Shan, S., Soltis, P. S., Soltis, D. E., and Yang, B. (2020). Considerations in Adapting CRISPR/Cas9 in Nongenetic Model Plant Systems. Appl. Plant Sci. 8 (1), e11314. doi:10.1002/aps3.11314

Shy, B. R., MacDougall, M. S., Clarke, R., and Merrill, B. J. (2016). Co-incident Insertion Enables High Efficiency Genome Engineering in Mouse Embryonic Stem Cells. Nucleic Acids Res. 44 (16), 7997-8010. doi:10.1093/nar/gkw685
Steinberger, A. R., Merino, W. O., Cahoon, R. E., Cahoon, E. B., and Lynch, D. V. (2021). Disruption of Long-chain Base Hydroxylation Alters Growth and Impacts Sphingolipid Synthesis in Physcomitrella Patens. Plant direct 5 (7), e336. doi:10.1002/pld3.336

Strepp, R., Scholz, S., Kruse, S., Speth, V., and Reski, R. (1998). Plant Nuclear Gene Knockout Reveals a Role in Plastid Division for the Homolog of the Bacterial Cell Division Protein FtsZ, an Ancestral Tubulin. Proc. Natl. Acad. Sci. 95 (8), 4368-4373. doi:10.1073/pnas.95.8.4368

Takechi, K., Nagase, H., Furuya, T., Hattori, K., Sato, Y., Miyajima, K., et al. (2021). Two Atypical ANGUSTIFOLIA without a Plant-specific C-terminus Regulate Gametophore and Sporophyte Shapes in the moss Physcomitrium (Physcomitrella) Patens. Plant J. 105 (5), 1390-1399. doi:10.1111/tpj.15121

Trogu, S., Ermert, A. L., Stahl, F., Nogué, F., Gans, T., and Hughes, J. (2020). Multiplex CRISPR-Cas9 Mutagenesis of the Phytochrome Gene Family in Physcomitrium (Physcomitrella) Patens. Plant Mol. Biol. 107, 327-336. doi:10.1007/s11103-020-01103-x

Vidali, L., and Bezanilla, M. (2012). Physcomitrella Patens: a Model for Tip Cell Growth and Differentiation. Curr. Opin. Plant Biol. 15 (6), 625-631. doi:10.1016/j.pbi.2012.09.008

Vidali, L., van Gisbergen, P. A. C., Guerin, C., Franco, P., Li, M., Burkart, G. M., et al. (2009). Rapid Formin-Mediated Actin-Filament Elongation Is Essential for Polarized Plant Cell Growth. Proc. Natl. Acad. Sci. 106 (32), 13341-13346. doi:10.1073/pnas.0901170106

Wiedemann, G., van Gessel, N., Köchl, F., Hunn, L., Schulze, K., Maloukh, L., et al. (2018). RecQ Helicases Function in Development, DNA Repair, and Gene Targeting in Physcomitrella Patens. Plant Cell 30 (3), 717-736. doi:10.1105/ tpc.17.00632

Wood, A. J., Lo, T.-W., Zeitler, B., Pickle, C. S., Ralston, E. J., Lee, A. H., et al. (2011). Targeted Genome Editing across Species Using ZFNs and TALENs. Science 333 (6040), 307. doi:10.1126/science.1207773

Wood, A. J., Oliver, M. J., and Cove, D. J. (2000). Bryophytes as Model Systems. The Bryologist 103 (1), 128-133. doi:10.1639/0007-2745(2000)103[0128:bams] 2.0.co;2

Yi, P., and Goshima, G. (2020). Transient Cotransformation of CRISPR /Cas9 and Oligonucleotide Templates Enables Efficient Editing of Target Loci in Physcomitrella Patens. Plant Biotechnol. J. 18 (3), 599-601. doi:10.1111/ pbi. 13238

Zimmer, A. D., Lang, D., Buchta, K., Rombauts, S., Nishiyama, T., Hasebe, M., et al. (2013). Reannotation and Extended Community Resources for the Genome of the Non-seed Plant Physcomitrella Patens Provide Insights into the Evolution of Plant Gene Structures and Functions. BMC Genomics 14, 498. doi:10.1186/ 1471-2164-14-498

Conflict of Interest: The author declares that the research was conducted in the absence of any commercial or financial relationships that could be construed as a potential conflict of interest.

Publisher's Note: All claims expressed in this article are solely those of the authors and do not necessarily represent those of their affiliated organizations, or those of the publisher, the editors and the reviewers. Any product that may be evaluated in this article, orclaim that may be made by its manufacturer, is not guaranteed or endorsed by the publisher.

Copyright () $2021 \mathrm{Zhu}$. This is an open-access article distributed under the terms of the Creative Commons Attribution License (CC BY). The use, distribution or reproduction in other forums is permitted, provided the original author(s) and the copyright owner(s) are credited and that the original publication in this journal is cited, in accordance with accepted academic practice. No use, distribution or reproduction is permitted which does not comply with these terms. 